\title{
Função e qualidade de vida de pacientes com fratura do planalto tibial operados com placa bloqueada ou convencional: estudo comparativo*
}

\section{Comparative Study of Function and Quality of Life in Patients with Fracture of the Tibial Plateau Operated with Locked or Conventional Plate}

\author{
Bruno Gonçalves Schröder e Souza ${ }^{10}$ Thiago Avelino Leite ${ }^{2}$ Tarsis Aparecido Bueno da Silva ${ }^{1}$ \\ Carlos Otavio Fabiano de Faria Candido ${ }^{1}$ Felipe Freesz de Almeida ${ }^{1}$ Valdeci Manoel de Oliveira ${ }^{1}$ \\ ${ }^{1}$ Faculdade de Ciências Médica e da Saúde de Juiz de Fora (Suprema), \\ Juiz de Fora, MG, Brasil \\ 2 Serviço de Ortopedia e Traumatologia, Hospital e Maternidade \\ Address for correspondence Bruno Gonçalves Schröder e Souza, MD, \\ Faculdade de Ciências Médica e da Saúde de Juiz de Fora, Juiz de Fora, \\ Therezinha de Jesus (HMTJ), Juiz de Fora, MG, Brasil \\ MG, Brazil (e-mail: brunogss01@yahoo.com.br).
}

Rev Bras Ortop 2019;54:37-44.

\begin{abstract}
Resumo
Objetivos Comparar resultados clínicos, funcionais e de qualidade de vida de pacientes com fratura do planalto tibial operados com placa bloqueada ou convencional e comparar os custos hospitalares dos implantes.

Métodos Estudo comparativo de coortes transversal, retrospectivo, em uma série consecutiva de pacientes com fratura do planalto tibial tratados cirurgicamente entre agosto de 2015 e junho de 2016. Foram excluídos: menores de 18 anos; indivíduos incapazes de responder os questionários ou de comparecer para reavaliação; politrauma-

Palavras-chave

- fraturas da tíbia

- placas ósseas

- qualidade de vida

- escore de Lysholm para joelho

- licença médica

- custos e análise de custo

- sistema único de saúde tizados ou com lesões associadas no mesmo membro; pacientes não tratados com placa ou conservadoramente. Os autores compararam os custos dos implantes, a qualidade de vida (SF-12), o escore de Lysholm, a escala visual de dor e os parâmetros clínicos e radiográficos. Resultados Foram observadas 45 fraturas no período, das quais 11 foram excluídas. Dos 34 pacientes, dois não compareceram à entrevista (seguimento de $94 \%$ ). 0 tempo de seguimento foi $15,1 \pm 4,8$ meses. O grupo $A$ (placa bloqueada) incluiu 22 pacientes (69\%), com custo hospitalar médio dos implantes de $R \$ 4.125,39$ ( $d p=R \$ 1.634,79 /$ paciente). $O$ grupo $B$ (placa convencional) incluiu dez pacientes (31\%), a um custo médio de $R \$ 438,53$ $(d p=R \$ 161,8 /$ paciente; $p<0,00001)$. Para os demais parâmetros avaliados, não foram observadas diferenças significativas entre os grupos, exceto por um maior degrau articular no grupo $A(2,7 \mathrm{~mm} \pm 3,3 \mathrm{~mm}$ vs. $0,5 \mathrm{~mm} \pm 1,6 \mathrm{~mm} ; \mathrm{p}=0,02 ; \mathrm{TE}=0,90)$.
\end{abstract}

\footnotetext{
Trabalho desenvolvido no Serviço de Ortopedia e Traumatologia, Hospital e Maternidade Therezinha de Jesus (HMTJ), Juiz de Fora, MG, Brasil. Publicado originalmente por Elsevier Editora Ltda. () 2018 Sociedade Brasileira de Ortopedia e Traumatologia.

(1D)Bruno Gonçalves Schröder e Souza's ORCID is https://orcid.org/ 0000-0002-8394-6840.
}

received

August 18, 2017

accepted

September 19, 2017

published online

January 12, 2018
DOI https://doi.org/

10.1016/j.rbo.2017.09.004. ISSN 0102-3616.
Copyright $\odot 2019$ by Sociedade Brasileira License terms de Ortopedia e Traumatologia. Published by Thieme Revnter Publicações Ltda, Rio de Janeiro, Brazil 


\begin{abstract}
Keywords

- tibial fractures

- bone plates

- quality of life

- Lysholm knee score

- sick leave

- cost and cost analysis

- unified health system

Objective To compare clinical, functional, and quality of life outcomes between patients with tibial plateau fractures operated with locked or conventional plates, and to compare the costs of these implants.

Methods This was a comparative cross-sectional study of a consecutive series of patients with tibial plateau fractures treated surgically from August 2015 to June 2016. Patients $<18$ years old, those unable to answer the questionnaires or to attend the outpatient reassessment, polytrauma patients, those with associated injuries on the ipsilateral limb, and patients who had not undergone treatment with bone plates were excluded. The present study compared the costs of the implants for the hospital, quality of life (with the 12-Item Short Form Health Survey [SF-12]), Lysholm score, pain scale, and clinical and radiological parameters.

Results A total of 45 patients with tibial plateau fractures were admitted, and 11 cases were excluded. Two cases were lost to follow-up; therefore, 32 remained for the analysis (94\%). The mean follow-up time was of 15.1 months (standard deviation $[S D]=4.8$ months). In group A (locked plates), there were 22 patients $(69 \%)$, at an average hospital cost of BRL 4,125.39/patient ( $S D=1,634.79 /$ patient) for the implants. In group B (conventional plates) there were 10 patients (31\%), at an average cost of BRL 438.53 (SD = 161.8/patient) ( $p<0.00001)$. For the other parameters, no differences were observed, except for a greater articular depression in group $A$ $(2.7 \mathrm{~mm} \pm 3.3 \mathrm{~mm}$ versus $0.5 \mathrm{~mm} \pm 1.6 \mathrm{~mm} ; p=0.02 ; \mathrm{TE}=0.90)$.

Conclusion The costs of locked implants for the treatment of tibial plateau fractures are significantly higher than those of conventional implants, without any clinical, quality of life, radiological, or functional advantages of the locked implants demonstrated in the present series.
\end{abstract}

Conclusão O custo dos implantes bloqueados para o tratamento das fraturas do planalto tibial é significativamente superior aos implantes convencionais, embora não tenham apresentado vantagem clínica, radiográfica, funcional ou de qualidade de vida, nos pacientes dessa amostra.

\section{Introdução}

As fraturas do planalto tibial representam 1 a $2 \%$ de todas as fraturas e aproximadamente $8 \%$ das fraturas em idosos. ${ }^{1,2}$ Um estudo de 2015 revelou que as fraturas do planalto tibial têm incidência de aproximadamente 10,3 casos por 100.000 habitantes. Acometem indivíduos de ambos os sexos, a maior incidência é em mulheres acima dos 50 anos e em homens abaixo dos 50 anos. $^{2}$ No Brasil, em 2015, foram feitas 11.071 cirurgias para o tratamento da fratura do planalto tibial pelo Sistema Único de Saúde (SUS), a um custo de R\$9.317.006,85 para o sistema. ${ }^{3}$

As fraturas de planalto tibial desviadas demandam tratamento cirúrgico. ${ }^{2}$ Vários fatores parecem inuenciar no resultado desse tratamento, entre eles a qualidade de redução, o tipo de fratura, a idade, lesões associadas e o tipo de implante. ${ }^{2}$ Embora possa existir variabilidade dos resultados conforme o tipo dos implantes e uma tendência atual na escolha de implantes bloqueados por cirurgiões ortopédicos, não está claro se o uso de um tipo específico de implante influencia no resultado clínico e funcional. ${ }^{4}$

Adicionalmente, existe uma preocupação crescente com o custo de implantes e o seu impacto nos sistemas de saúde tanto públicos quanto privados..$^{5}$ Para que estudos de custoefetividade possam responder se o uso ou a adoção de determinado tipo de implante é adequado, são necessários estudos que comparem a efetividade de diferentes implantes e considerem diversos fatores, como dor, função, qualidade de vida e o tempo de afastamento de trabalho. ${ }^{5,6}$

Em nosso meio, não encontramos estudos comparativos que ajudem a esclarecer as possíveis diferenças entre as fraturas de planaltos tibiais tratadas cirurgicamente com a placa bloqueada (-Fig. 1) ou placas convencionais (-Fig. 2). 0 objetivo deste estudo é comparar resultados clínicos, funcionais e de qualidade de vida entre os pacientes com fratura do planalto tibial operados com placa bloqueada ou convencional. Paralelamente objetivamos comparar os custos hospitalares dos diferentes tipos de implantes na amostra.

\section{Material e Métodos}

Trata-se de um estudo comparativo de coortes retrospectivo em uma série consecutiva de pacientes com fratura do planalto tibial, tratados cirurgicamente com placa bloqueada (Grupo A) ou convencional (Grupo B), de agosto de 2015 a 


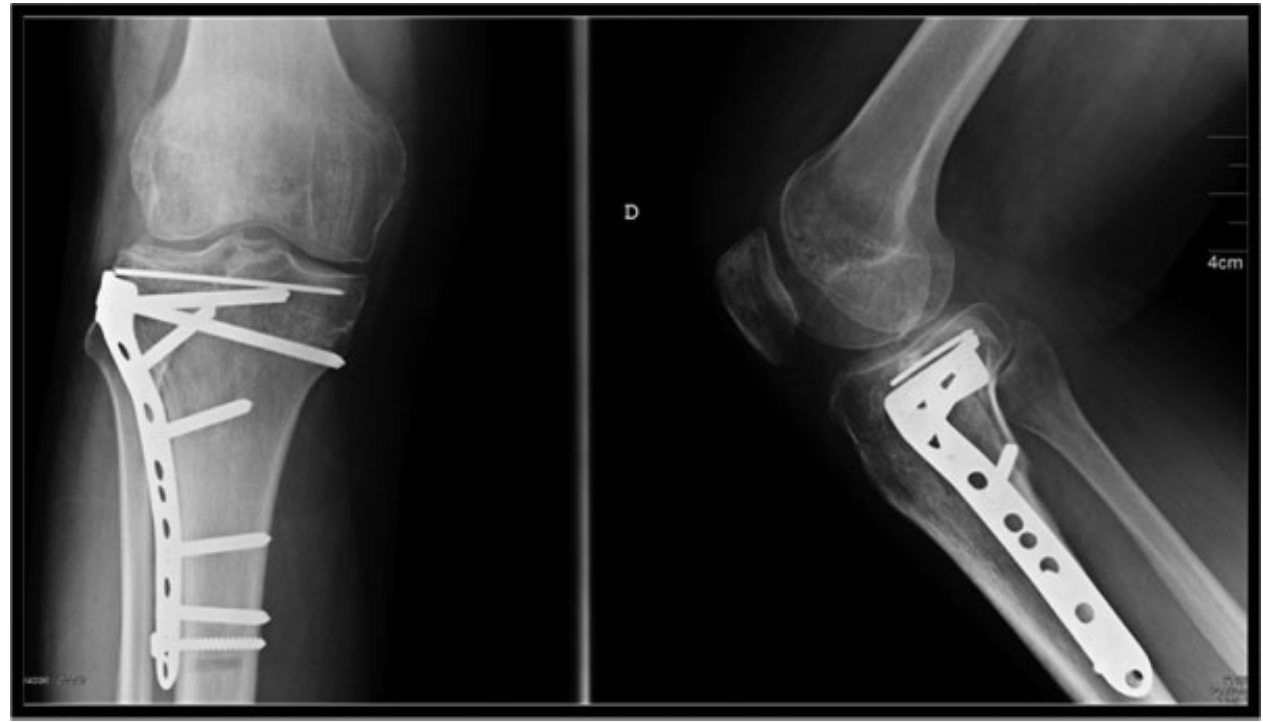

Fig. 1 Osteossíntese por placa bloqueada, visualizada em radiografias em AP e Perfil.

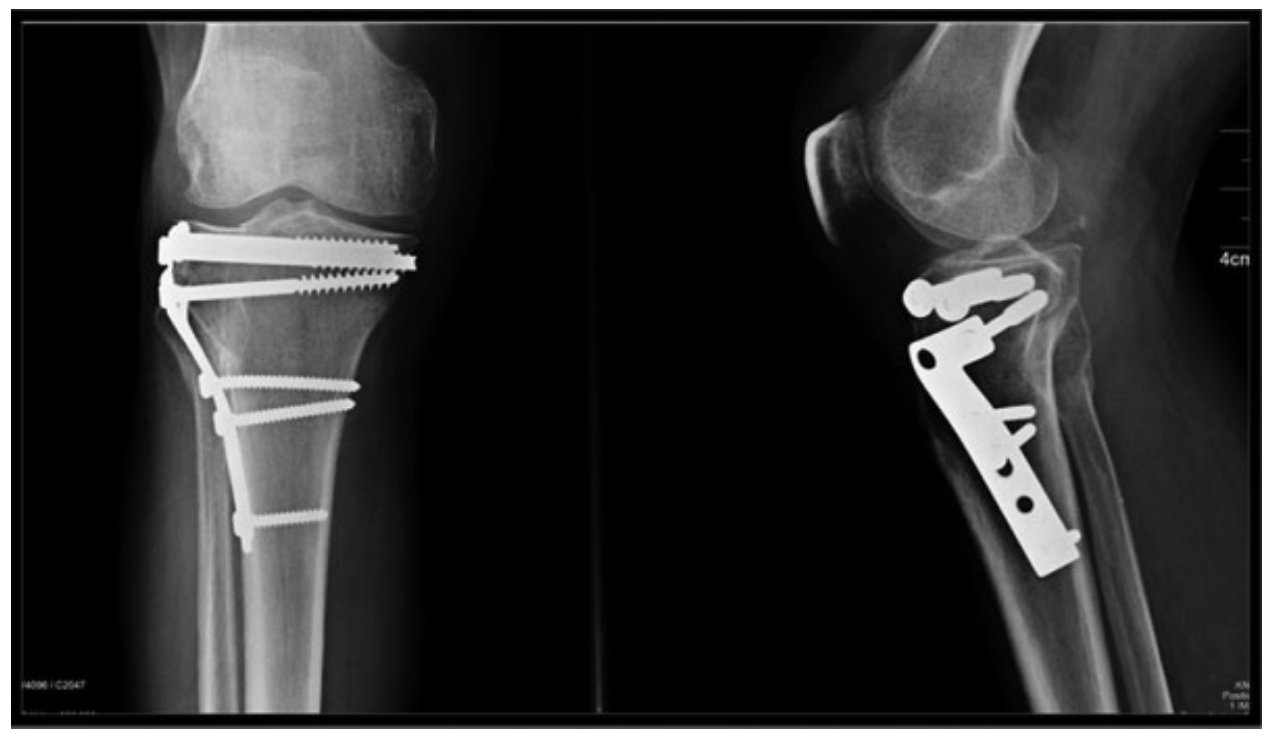

Fig. 2 Osteossíntese por placa convencional, visualizada em radiografias em AP e Perfil.

junho de 2016, em um hospital terciário de ensino que atende exclusivamente pacientes do Sistema Único de Saúde (SUS).

Foram incluídos todos os pacientes internados por fratura de planalto tibial, no mesmo hospital, de agosto de 2015 a junho de 2016. Foram excluídos: pacientes menores de idade ( $<18$ anos), incapazes de responder os questionários de avaliação funcional ou de comparecer para reavaliação ambulatorial; indivíduos politraumatizados ou com lesões associadas no mesmo membro ou em outra região anatômica que afetavam de maneira decisiva a reabilitação funcional do membro; pacientes tratados sem placa; pacientes em que foi feito tratamento conservador. Os casos excluídos e os motivos são apresentados nos resultados.

O estudo foi aprovado pelo Comitê de Ética em Pesquisa sob o número 65959717.9.0000.5103 e todos os participantes assinaram o Termo de Consentimento Livre e Esclarecido.
Os participantes foram submetidos prospectivamente, pelo mesmo avaliador, à avaliação clínica e funcional com questionários padronizados e validados. A análise das radiografias pósoperatórias também foi feita pelo mesmo avaliador, no momento do retorno. A qualidade de vida no momento da consulta de seguimento também foi registrada. Os prontuários físicos e eletrônicos dos pacientes e todos os exames de imagem disponíveis foram avaliados para coleta de dados referentes à demografia, eventos relacionados ao trauma, tipo de fratura, material usado e custos dos implantes para o hospital. Foram registradas também a função neurológica antes e após o ato cirúrgico, dados sobre infecção, mas com a ferida operatória, retardo de consolidação e outras complicações.

Os parâmetros: tipo de fratura pela classificação de Schatzker, qualidade da redução (presença de desvios articulares em milímetros, desvios angulares nas radiografias em AP e perfil), número de parafusos em cada placa, número de parafusos fora da placa, radiografia inicial, pós-operatória 
imediata e atual, foram colhidos e anotados em formulário padrão. Nas radiografias atuais também foram avaliadas o estado da consolidação, a presença de soltura ou falha do implante, desvio residual e sinais de artrose pela classificação de Alback. ${ }^{7,8}$ Os questionários aplicados no retorno foram: Escala Visual Analógica de dor (EVA), questionário clínico funcional Lysholm ${ }^{9}$ e 12-Item Short Form Health Survey - SF12; ${ }^{10}$ e exame clínico padronizado avaliou o arco de movimento do joelho, déficit de flexão e a extensão e situação da ferida operatória.

\section{Análise estatística}

As variáveis quantitativas foram descritas pela média e pelo desvio-padrão e as variáveis qualitativas por frequência absoluta e porcentagens. Para testar diferenças entre os grupos em relação às variáveis quantitativas, foi usado o teste $t$ de Student para amostras independentes ou o teste U de Mann-Whitney, quando apropriado. $\mathrm{O}$ tamanho do efeito foi avaliado pelo d de Cohen, foi adotada a seguinte classificação para interpretação: $0,20-0,49$, pequeno; 0,50-0,79, moderado; $\geq 0,80$, elevado. ${ }^{11}$ Para testar diferenças entre proporções, usou-se o teste exato de Fisher, foi usado o V de Cramer como medida do tamanho do efeito, foi adotada a seguinte classificação para interpretação: $0,10-0,29$, pequeno; 0,30-0,49, moderado; $\geq 0,50$, elevado. ${ }^{11}$ Calculou-se a razão de chances (OR) e o intervalo de confiança de 95\% (IC95\%). Todas as análises foram feitas no software estatístico IBM SPSS versão 20.0 (IBM Corp., Armonk, NY). O valor de $\mathrm{p}<0,05$ foi adotado para significância estatística.

\section{Resultados}

Entre agosto de 2015 e junho de 2016 foram internadas 45 fraturas do planalto tibial, que foram incluídas no estudo. Houve 11 exclusões: sete devido a tratamento cirúrgico que usou apenas parafusos, uma por fratura côndilo femoral associada, uma por ter sido tratada conservadoramente, duas por dados insuficientes em prontuários. Da amostra inicial de 34 pacientes, dois não compareceram à entrevista, resultou numa amostra de 32 pacientes (seguimento de 94\%) (-Fig. 3). O tempo médio de seguimento foi de $15,1 \pm 4,8$ meses (de oito a 26).

Os dados demográficos relacionados aos grupos estudados e suas fraturas estão expressos na - Tabela 1. Os grupos A e B se mostraram similares em relação a idade, sexo, lado acometido, mecanismo e tipo de fratura, tempo até a cirurgia e tempo de seguimento $(p>0,05)$. No entanto, parece existir uma relação entre a gravidade da fratura e o tipo de implante ( $\mathrm{p}=0,12 ; \mathrm{V}=0,31$ ) de magnitude moderada, sob o ponto de vista prático. Ao considerar o tipo de implante como desfecho e a gravidade da fratura como fator de exposição, observa-se que $80 \%$ dos pacientes que apresentam Schatzker 5 e 6 têm implante com placa bloqueada contra $50 \%$ dos pacientes com Schatzker 1 a 4 . Isso significa que a chance de os cirurgiões escolherem um implante com placa bloqueada é quatro vezes maior nos pacientes Schatzker 5 e 6 do que nos pacientes com Schatzker 1 a 4 (OR $($ IC95\% $)=4,00$ (0,83-19,32). Embora não significativo estatisticamente, esse resultado, sob o ponto de vista prático, apresenta um efeito de elevada magnitude conforme a classificação do V de Cramer.

No grupo A houve 22 pacientes (69\%) operados com placa bloqueada, com um custo hospitalar dos implantes de R\$ 90.758,6 (média $=\mathrm{R} \$ 4.125,39 \pm 1.634,79 /$ paciente). No grupo $\mathrm{B}$, houve 10 pacientes (31\%) operados com placa convencional com um custo hospitalar dos implantes de $\mathrm{R} \$$ 4.385,36 (média $=\mathrm{R} \$ 438,53 \pm 161,8 /$ paciente) - - Fig. 4 . A diferença dos valores nos custos hospitalares do implante foi significativa a um $\mathrm{p}<0,00001$.

Os dados relacionados à internação e aos procedimentos cirúrgicos são apresentados na - Tabela 2. Não foram observadas diferenças estatisticamente significantes entre os grupos quanto ao tempo de internação, número de placas usadas e número de vias de acesso, assim como em relação a complicações e necessidade de reoperação ( $p>0,05)$.

A - Tabela 3 apresenta os dados da avaliação radiográfica. Não foram encontradas diferenças estatisticamente significantes em relação ao tempo de consolidação, aos ângulos tibiais no pós-operatório imediato e sinais de gonartrose (p $>0,05$ ). Porém, o Grupo Bloqueado (grupo A) apresentou maior desvio articular comparado com o Grupo Convencional (grupo B) $(2,7 \mathrm{~mm} \pm 3,3 \mathrm{~mm}$ vs. $0,5 \mathrm{~mm} \pm 1,6 \mathrm{~mm}$, $\mathrm{p}=0,02 ; \mathrm{TE}=0,90)$. Sob o ponto de vista prático, a diferença encontrada foi de elevada magnitude.

A - Tabela 4 apresenta os resultados clínicos, funcionais e de qualidade de vida. Não foram observadas diferenças estatisticamente significantes na avaliação funcional, dor e qualidade de vida ( $\mathrm{p}>0,05)$.

Na - Tabela 5 são observados os impactos socioeconômicos da cirurgia. Não foram observadas diferenças estatisticamente significantes entre os grupos em relação ao tempo médio de afastamento e a frequência de retorno ao trabalho ( $\mathrm{p}>0,05)$.

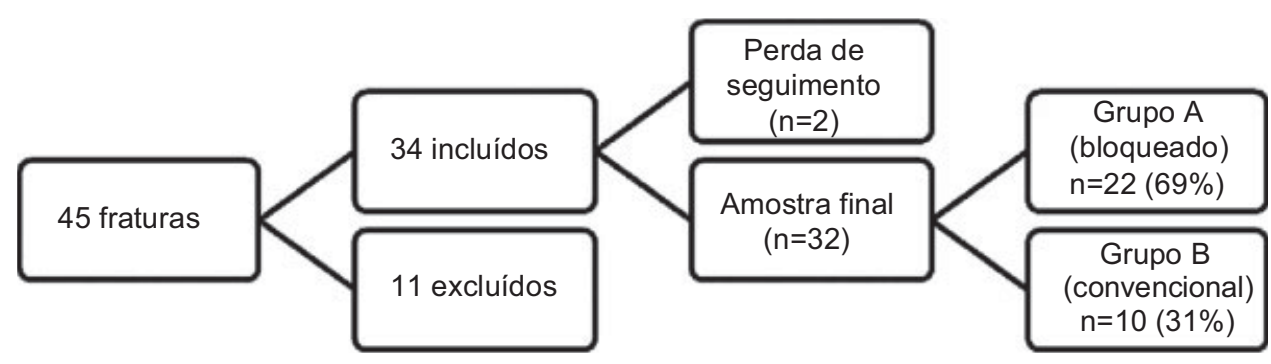

Fig. 3 Amostra de seguimento do estudo. 
Tabela 1 Características demográficas e do tipo de fratura em pacientes com fratura do planalto tibial operados com placa bloqueada ou convencional

\begin{tabular}{|c|c|c|c|c|}
\hline Fator & $\begin{array}{l}\text { Grupo Bloqueado } \\
(\mathrm{n}=22)\end{array}$ & $\begin{array}{l}\text { Grupo Convencional } \\
(n=10)\end{array}$ & Todos $(n=32)$ & p-valor \\
\hline Idade (anos) & $45,9 \pm 12,8$ & $46,3 \pm 16,4$ & $46,1 \pm 13,7$ & $0,95^{\mathrm{a}}$ \\
\hline \multicolumn{5}{|l|}{ Sexo } \\
\hline Masculino & $14(64 \%)$ & $09(90 \%)$ & $23(72 \%)$ & $0,21^{\mathrm{b}}$ \\
\hline Feminino & $08(36 \%)$ & $01(10 \%)$ & $09(28 \%)$ & \\
\hline \multicolumn{5}{|l|}{ Lado acometido } \\
\hline Esquerdo & $12(54 \%)$ & $04(40 \%)$ & $16(50 \%)$ & $0,7^{\mathrm{b}}$ \\
\hline Direito & $10(46 \%)$ & $06(60 \%)$ & $16(50 \%)$ & \\
\hline \multicolumn{5}{|l|}{ Mecanismo } \\
\hline Moto & $10(46 \%)$ & $05(50 \%)$ & $15(47 \%)$ & $0,37^{\mathrm{b}}$ \\
\hline Atropelamento & $03(14 \%)$ & $04(40 \%)$ & 07 (22\%) & \\
\hline Queda de altura & $02(09 \%)$ & $00(0 \%)$ & $02(6,3 \%)$ & \\
\hline Automóvel & $01(4 \%)$ & $01(10 \%)$ & $02(6,3 \%)$ & \\
\hline Queda da própria altura & $04(18 \%)$ & $00(0 \%)$ & $04(12,5 \%)$ & \\
\hline Outros & $02(09 \%)$ & $00(0 \%)$ & $02(6,3 \%)$ & \\
\hline \multicolumn{5}{|l|}{ Tipo } \\
\hline Unicondilar (Schatzker 1-4) & $06(28 \%)$ & $06(60 \%)$ & $12(37,5 \%)$ & $0,12^{\mathrm{b}}$ \\
\hline Bicondilar (Schatzker 5-6) & $16(72 \%)$ & $04(40 \%)$ & $20(62,5 \%)$ & \\
\hline Tempo até a cirurgia (dias) & $13,5 \pm 7,8$ & $12,5 \pm 7,1$ & $13,2 \pm 7,5$ & $0,73^{\mathrm{a}}$ \\
\hline Tempo de seguimento (meses) & $15,2 \pm 5,2$ & $14,8 \pm 3,8$ & $15,1 \pm 4,8$ & $0,84^{\mathrm{a}}$ \\
\hline
\end{tabular}

${ }^{a}$ Valores calculados pelo teste $t$ de Student.

bValores calculados pelo teste exato de Fisher.

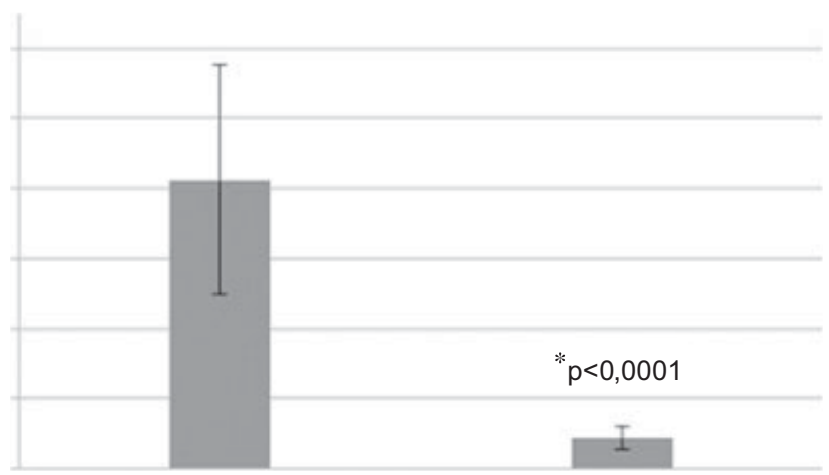

Fig. 4 Custo médio dos implantes em pacientes com fratura do planalto tibial operados com placa bloqueada ou convencional ( ${ }^{*}$ diferença estatisticamente significante, $p<0.05$ ).

\section{Discussão}

Nosso estudo confirmou a impressão de que existe uma tendência dos cirurgiões de escolherem placas bloqueadas em padrões de fraturas mais graves. Embora não tenha alcanç ado significância estatística, esse dado corrobora os achados descritos em uma recente metanálise. ${ }^{12}$ Naquele estudo, não foram observadas diferenças entre os resultados clínicos, funcionais e de qualidade de vida entre os pacientes opera- dos com diferentes tipos de implante. Nosso estudo chegou à mesma conclusão, exceto com relação aos custos.

Os custos diretos para o hospital das placas bloqueadas foram significativamente maiores do que os da placa convencional (aproximadamente dez vezes) ( $\mathrm{p}<0.0001$ ). Esse achado também foi relatado em outros tipos de fraturas. ${ }^{13,14}$ Um estudo que comparou o tratamento cirúrgico das fraturas de olécrano mostrou um aumento médio de US\$ $1.263,50$ no custo dos pacientes operados com placa bloqueada. ${ }^{13}$ No joelho, essa diferença nos gastos também foi encontrada, o que reforça a necessidade de discutir a real indicação desse tipo de implante. $^{14}$

No âmbito do SUS, no qual a tabela de procedimentos é extremamente defasada e contempla atualmente essas fraturas com uma verba de $\mathrm{R} \$ 252,08$ para o pagamento do hospital, observamos ser inviável o uso indiscriminado desses implantes, pois o déficit por paciente (considerando apenas o custo do implante) seria de $\mathrm{R} \$ 3.873,81$. De fato, nosso estudo prova que o valor pago pelo sistema não cobre sequer o custo dos implantes convencionais. Portanto, no cenário atual, implantes especiais só deveriam ser usados nos casos em que forem realmente imprescindíveis, apesar de a literatura atual não apresentar indicações absolutas para uso de implantes bloqueados, ${ }^{6,14}$ o que reforça o hiato quanto o uso desses implantes no âmbito do SUS. 
42 Tratamento comparativo de fratura do planalto tibial Schröder e Souza et al.

Tabela 2 Características da internação e cirurgia em pacientes com fratura do planalto tibial operados com placa bloqueada ou convencional

\begin{tabular}{|c|c|c|c|c|}
\hline Fator & $\begin{array}{l}\text { Grupo Bloqueado } \\
(n=22)\end{array}$ & $\begin{array}{l}\text { Grupo Convencional } \\
(n=10)\end{array}$ & $\begin{array}{l}\text { Todos } \\
(n=32)\end{array}$ & p-valor \\
\hline Tempo de internação (dias) & $17,9 \pm 10,5$ & $13,7 \pm 7,0$ & $16,6 \pm 9,6$ & $0,25^{a}$ \\
\hline \multicolumn{5}{|l|}{ Número de placas } \\
\hline Uma & $13(59 \%)$ & $07(70 \%)$ & $20(62 \%)$ & $0,7^{\mathrm{a}}$ \\
\hline Duas ou mais & $09(41 \%)$ & $03(30 \%)$ & $12(38 \%)$ & \\
\hline \multicolumn{5}{|l|}{ Número de vias de acesso } \\
\hline Uma & $13(59 \%)$ & $07(70 \%)$ & $20(62 \%)$ & $0,42^{\mathrm{a}}$ \\
\hline Duas & $09(41 \%)$ & $03(30 \%)$ & $12(38 \%)$ & \\
\hline \multicolumn{5}{|l|}{ Complicações } \\
\hline Sim & $01(4,5 \%)$ & $01(10 \%)$ & $02(6,3 \%)$ & $0,53^{b}$ \\
\hline Não & $21(95,5 \%)$ & 09 (90\%) & $30(93,7 \%)$ & \\
\hline \multicolumn{5}{|l|}{ Reoperações } \\
\hline Sim & $03(13,5 \%)$ & $00(0 \%)$ & $03(9,4 \%)$ & $0,53^{b}$ \\
\hline Não & $19(86,5 \%)$ & $10(100 \%)$ & $29(90,6 \%)$ & $0,25^{\mathrm{a}}$ \\
\hline
\end{tabular}

${ }^{a}$ Valores calculados pelo teste t de Student.

${ }^{b}$ Valores calculados pelo teste exato de Fisher.

Tabela 3 Avaliação radiográfica de pacientes com fratura do planalto tibial operados com placa bloqueada ou convencional

\begin{tabular}{|l|l|l|l|l|}
\hline Fator & $\begin{array}{l}\text { Grupo Bloqueado } \\
(\mathbf{n = 2 2})\end{array}$ & $\begin{array}{l}\text { Grupo Convencional } \\
(\mathbf{n}=\mathbf{1 0})\end{array}$ & $\begin{array}{l}\text { Todos } \\
(\mathbf{n}=32)\end{array}$ & p-valor \\
\hline Tempo de consolidação (dias) & $71,9 \pm 23,1$ & $68,5 \pm 16,1$ & $70,8 \pm 20,9$ & $0,67^{\mathrm{a}}$ \\
\hline Desvio articular & $2,7 \pm 3,3$ & $0,5 \pm 1,6$ & $2,0 \pm 3,1$ & $0,02^{\mathrm{b}, \mathrm{c}}$ \\
\hline $\begin{array}{l}\text { Ângulo articular da tíbia } \\
\text { (anteroposterior) (o) }\end{array}$ & $3,5 \pm 4,3$ & $2,7 \pm 4,0$ & $3,2 \pm 4,2$ & $0,19^{\mathrm{a}}$ \\
\hline Sinais de gonartrose & & & $11(35 \%)$ & $0,1^{\mathrm{d}}$ \\
\hline Sim & $10(45 \%)$ & $01(10 \%)$ & $21(65 \%)$ & $0,67^{\mathrm{a}}$ \\
\hline Não & $12(65 \%)$ & $09(90 \%)$ & $0,02^{\mathrm{b}, \mathrm{c}}$ \\
\hline
\end{tabular}

${ }^{a}$ Valores calculados pelo teste t de Student.

balores calculados pelo teste de Mann-Whitney.

'Diferença estatisticamente significante, $\mathrm{p}<0,05$ pelo teste de Mann-Whitney.

dValores calculados pelo teste exato de Fisher.

Em nosso estudo, ao avaliar os pacientes operados em ambos os grupos, observamos que os resultados clínicos, funcionais e radiográficos foram semelhantes, assim como em outros estudos na literatura. ${ }^{14,15}$ Em uma comparação de fratura tipo Schatzker II, ${ }^{14}$ não foram observadas diferenças entre os dois tipos de placa, a não ser o custo. No entanto, aquele estudo não avaliou a qualidade de vida ou o afastamento do trabalho. Nosso estudo mostra que além de não haver diferença nos parâmetros citados inicialmente, o uso de placas bloqueadas também não trouxe diferenças significativas na qualidade de vida ou no tempo de afastamento do trabalho. $\mathrm{O}$ número limitado de nossa amostra não permitiu, entretanto, uma estratificação para identificar subgrupos específicos de pacientes que pudessem potencialmente se beneficiar dos implantes bloqueados.
As características demográficas de nossos pacientes se assemelham às de outros estudos. Encontramos que a principal causa de fraturas foram os acidentes de trânsito $(76 \%$, ) e que a média de idade foi de 46 anos. Em outro estudo nacional ${ }^{16} \mathrm{o}$ principal mecanismo de trauma foi o mesmo e a média de idade foi de 45,5 anos, o que ajuda na validação externa dos nossos resultados. A implicação desses achados é que essas fraturas têm relevante impacto socioeconômico, pois acometem geralmente adultos em idade produtiva e provocam tempos prolongados de afastamento do trabalho. Observamos uma taxa de retorno ao trabalho de $72,7 \%$ em média até o fim do nosso seguimento, o que reforça esses achados. De fato, um estudo prospectivo aleatorizado multicêntrico demonstrou que, após 24 meses, apenas 30\% dos pacientes consideraram que conseguiram retornar plenamente ao seu nível prévio de atividade. ${ }^{17}$ 
Tabela 4 Resultados clínicos, funcionais e de qualidade de vida de pacientes com fratura do planalto tibial operados com placa bloqueada ou convencional

\begin{tabular}{|c|c|c|c|c|}
\hline Fator & $\begin{array}{l}\text { Grupo Bloqueado } \\
(\mathrm{n}=22)\end{array}$ & $\begin{array}{l}\text { Grupo Convencional } \\
(\mathrm{n}=10)\end{array}$ & Todos $(n=32)$ & p-valor \\
\hline \multicolumn{5}{|l|}{ EVA } \\
\hline Leve & $13(57 \%)$ & $07(70 \%)$ & $20(62 \%)$ & $0,87^{\mathrm{a}}$ \\
\hline Moderado & $05(24 \%)$ & $02(20 \%)$ & 07 (22\%) & \\
\hline Intenso & $04(19 \%)$ & $01(20 \%)$ & $05(16 \%)$ & \\
\hline \multicolumn{5}{|l|}{ SF-12 } \\
\hline $\mathrm{SF} \mathrm{m}$ & $21,4 \pm 6,2$ & $24,2 \pm 3,6$ & $22,3 \pm 5,6$ & $0,13^{\mathrm{a}}$ \\
\hline SF f & $16,3 \pm 3,7$ & $16,8 \pm 4,2$ & $16,5 \pm 3,8$ & $0,74^{\mathrm{a}}$ \\
\hline $\mathrm{SF} \mathrm{t}$ & $37,8 \pm 8,3$ & $41,0 \pm 7,5$ & $38,8 \pm 8,1$ & $0,3^{\mathrm{a}}$ \\
\hline \multicolumn{5}{|l|}{ Lysholm } \\
\hline Excelente e bom & $12(54 \%)$ & 07 (70\%) & 19 (59\%) & $0,7^{\mathrm{a}}$ \\
\hline Regular e ruim & $10(46 \%)$ & $03(30 \%)$ & $13(41 \%)$ & \\
\hline Mobilidade ADM & $121,4 \circ \pm 21,9 \circ$ & $129,0 \circ \pm 19,1 \circ$ & $123,8 \pm 21,1$ & $0,35^{\mathrm{b}}$ \\
\hline Déficit de flexão & $12,3 \circ \pm 17,2 \circ$ & $9,0 \circ \pm 15,9$ & $11,3 \pm 16,6$ & $0,61^{\mathrm{b}}$ \\
\hline Déficit de extensão & $4,6 \circ \pm 6,7 \circ$ & $2,0 \circ \pm 4,2 \circ$ & $3,8 \pm 6,1$ & $0,28^{\mathrm{b}}$ \\
\hline
\end{tabular}

Abreviações: EVA, Escala Visual Analógica de Dor; SF12, qualidade de vida.

a Valores calculados pelo teste exato de Fisher.

balores calculados pelo teste $t$ de Student.

Tabela 5 Impacto socioeconômico em pacientes com fratura do planalto tibial operados com placa bloqueada ou convencional

\begin{tabular}{|c|c|c|c|c|}
\hline Fator & $\begin{array}{l}\text { Grupo Bloqueado } \\
(\mathrm{n}=22)\end{array}$ & $\begin{array}{l}\text { Grupo Convencional } \\
(\mathrm{n}=10)\end{array}$ & $\begin{array}{l}\text { Todos } \\
(\mathbf{n}=32)\end{array}$ & p-valor \\
\hline \multicolumn{5}{|l|}{ Afastado do trabalho } \\
\hline Sim & $14(63 \%)$ & $8(80 \%)$ & $22(68 \%)$ & $0,78^{a}$ \\
\hline Não & $2(9 \%)$ & $0(0 \%)$ & $2(06 \%)$ & \\
\hline Aposentado & $6(28 \%)$ & $2(20 \%)$ & $8(26 \%)$ & \\
\hline Tempo de afastamento (dias) & $211,3 \pm 126,5$ & $174,3 \pm 97,1$ & $196,1 \pm 113,5$ & $0,53^{b}$ \\
\hline Conseguiu retornar ao trabalho (sim/\%) & $8(50 \%)$ & $7(87,5 \%)$ & $15(62,5 \%)$ & $0,39^{\mathrm{a}}$ \\
\hline
\end{tabular}

${ }^{\mathrm{a}}$ Valores calculados pelo teste exato de Fisher.

balores calculados pelo teste t de Student.

Alguns estudos demonstram que o principal fator relacionado ao prognóstico dessas fraturas é a qualidade da redução articular e que desvios articulares maiores do que $2,5 \mathrm{~mm}$ se relacionam a piores resultados. ${ }^{15}$ Em nosso estudo, a média de desvio foi considerada aceitável $(1,9$ $\mathrm{mm}$ ), embora no grupo de placas bloqueadas a média tenha ficado marginalmente acima desse valor. De fato, houve uma diferença significativa entre os desvios observados nos grupos bloqueado e convencional, em nosso estudo, o que favorece os implantes convencionais. Esse achado repete os de Abghari et al., ${ }^{14}$ que atribuíram essa pior redução à técnica indireta da redução nos casos operados por via minimamente invasiva (comum nos implantes bloqueados). Além disso, em nossa casuística, uma prevalência maior de fraturas bicondilares no grupo da placa bloqueada (não significante) poderia ter alguma relação com esse achado.
Em nosso estudo obtivemos 11 pacientes com sinais de gonartrose (34\%) após 15 meses de seguimento em média. Essa incidência é semelhante a estudo publicado em 2016, ${ }^{18}$ apesar de o tempo de seguimento não ser suficiente para identificar todos os casos que irão evoluir dessa forma. ${ }^{19}$

0 presente estudo tem uma série de limitações. Em primeiro lugar, a sua natureza retrospectiva impediu a aleatorização dos pacientes e melhor homogeneidade dos grupos. No entanto, por avaliar os procedimentos feitos no contexto real, os achados têm maior probabilidade de ter validade externa e representar medidas de efetividade dos tratamentos. Outro ponto negativo foi o número limitado de pacientes, que impediu a exploração de subgrupos de pacientes, para os quais os potenciais benefícios de implantes bloqueados poderiam ser identificados. No entanto, a análise estatística mostrou uma baixa correlação dos escores EVA e Lysholm com o tipo de implante, o que indica que a 
falta de significância encontrada não está relacionada a erro tipo beta (falta de poder ou amostra insuficiente). Não obstante, os achados de nosso estudo demonstram que, para realidade de nosso hospital, os custos dos implantes bloqueados para fraturas do planalto tibial são proibitivos e que, a princípio, o uso de implantes convencionais não provocou resultados radiográficos, clínicos, funcionais ou de qualidade de vida, inferiores aos implantes especiais bloqueados. Isso não significa, no entanto, que esse tipo de implante seja prescindível, uma vez que pode ser necessário em indicações cirúrgicas específicas e parece ser bem aceito entre os cirurgiões.

\section{Conclusão}

Observamos que os custos dos implantes bloqueados para o tratamento das fraturas do planalto tibial são significativamente superiores aos dos implantes convencionais, sem, no entanto, apresentar qualquer vantagem clínica, radiográfica, funcional ou de qualidade de vida, nos pacientes de nossa amostra.

\section{Conflitos de interesse}

Os autores declaram não haver conflitos de interesse.

\section{Referências}

1 Jacofsky DJ, Haidukerwych GJ. Tibia plateau fractures. In: Scott WN, ed. Insall \& Scott surgery of the knee. Philadelphia: Churchill Livingstone; 2006:1133-46

2 Elsoe R, Larsen P, Nielsen NP, Swenne J, Rasmussen S, Ostgaard SE. Population-based epidemiology of tibial plateau fractures. Orthopedics 2015;38(9):e780-6

3 Departamento de Informática do SUS [Base de dados online]. Brasília: MS - Ministério da Saúde 2017 [acesso em 2017 jun 21] Disponível em: http://tabnet.datasus.gov.br/cgi/tabcgi.exe?sih/ cnv/qiuf.def

4 Pelser PC. Controversies in the management of tibial plateau fractures. SA Orthop J 2010;9(3):75-82

5 Logan C, Hess A, Kwon JY. Damage control orthopaedics: Variability of construct design for external fixation of the lower extremity and implications on cost. Injury 2015;46(8):1533-8
6 Russell N, Tamblyn P, Jaarsma R. Tibial plateau fractures treated with plate fixation: to lock or not to lock. Eur J Orthop Surg Traumatol 2009;19(2):75-82

7 Markhardt BK, Gross JM, Monu JUV. Schatzker classification of tibial plateau fractures: use of CT and MR imaging improves assessment. Radiographics 2009;29(2):585-97

8 Petersson IF, Boegård T, Saxne T, Silman AJ, Svensson B. Radiographic osteoarthritis of the knee classified by the Ahlbäck and Kellgren \& Lawrence systems for the tibiofemoral joint in people aged 35-54 years with chronic knee pain. Ann Rheum Dis 1997;56 (8):493-6

9 Peccin MS, Ciconelli R, Cohen M. Questionário específico para sintomas do joelho "Lysholm Knee Scoring Scale": tradução e validação para a língua portuguesa. Acta Ortop Bras 2006;14(5): 268-72

10 Silveira MF, Almeida JC, Freire RS, Haikal DS, Martins AE. Propriedades psicométricas do instrumento de avaliação da qualidade de vida: 12 -item health survey (SF-12). Cien Saude Colet 2013;18(7):1923-31

11 Cohen J. A power primer. Psychol Bull 1992;112(1):155-9

12 McNamara IR, Smith TO, Shepherd KL, Clark AB, Nielsen DM, Donell $S$, et al. Surgical fixation methods for tibial plateau fractures. Cochrane Database Syst Rev 2015;(9):CD009679

13 DelSole EM, Egol KA, Tejwani NC. Construct choice for the treatment of displaced, comminuted olecranon fractures: are locked plates cost effective? Iowa Orthop J 2016;36:59-63

14 Abghari M, Marcano A, Davidovitch R, Konda SR, Egol KA. Are locked plates needed for split depression tibial plateau fractures? J Knee Surg 2016;29(6):482-6

15 Singleton N, Sahakian V, Muir D. Outcome after tibial plateau fracture: How important is restoration of articular congruity? J Orthop Trauma 2017;31(3):158-63

16 Albuquerque RP, Hara R, Prado J, Schiavo L, Giordano V, do Amaral NP. Epidemiological study on tibial plateau fractures at a level I trauma center. Acta Ortop Bras 2013;21(2):109-15

17 Canadian Orthopaedic Trauma Society. Open reduction and internal fixation compared with circular fixator application for bicondylar tibial plateau fractures. Results of a multicenter, prospective, randomized clinical trial. J Bone Joint Surg Am 2006;88(12):2613-23

18 Rohra N, Suri HS, Gangrade K. Functional and radiological outcome of Schatzker type V and VI tibial plateau fracture treatment with dual plates with minimum 3 years follow-up: a prospective study. J Clin Diagn Res 2016;10(5):RC05-10

19 Mattiassich G, Foltin E, Scheurecker G, Schneiderbauer A, Kröpfl A, Fischmeister M. Radiographic and clinical results after surgically treated tibial plateau fractures at three and twenty two years postsurgery. Int Orthop 2014;38(3):587-94 